\title{
Molecular Dynamics in Cyclic Olefin Copolymer
}

\author{
M. Makrocka-Rydzyk, B. Orozbaev, G. Nowaczyk, \\ S. GŁowinkowski And S. JurgA* \\ Department of Macromolecular Physics, Adam Mickiewicz University \\ Umultowska 85, 61-614 Poznań, Poland
}

\begin{abstract}
Nuclear magnetic resonance, broadband dielectric spectroscopy and dynamic-mechanical thermal analysis were employed to study molecular dynamics of ethylene-norbornene copolymer. The analysis of experimental data indicates existence of three motional processes denoted as $\alpha, \beta$, and $\gamma$ in order of decreasing temperature. The $\alpha$ relaxation is related to the dynamic glass transition, while the $\beta$ relaxation, observed only for the untreated sample, is assigned to short range segmental motions involving norbornene units. The $\gamma$ relaxation is due to very local motions of ethylene units e.g. trans-gauche isomerization, similar to those responsible for $\gamma$ relaxation in polyethylene. The rate of motion accountable for $\gamma$ process, follows the Vogel-Fulcher-Tammann equation, similarly to $\alpha$ transition, indicating cooperative nature of the motion.
\end{abstract}

PACS numbers: 36.20.Ey, 76.60.--k, 77.22.Gm, 83.85.Vb, 82.35.Jk

\section{Introduction}

Knowledge of the relation between structure and molecular dynamics in polymers is very important to understand their physical properties. One of the ways to obtain polymer with desired properties is copolymerization of composites having different dynamical properties. The cyclic olefin copolymers (COC) are polymers, which combine a flexibility of olefins with strength and rigidity of norbornene. Due to their excellent transparency, low density, and thermal and chemical resistance they are useful materials in optic industry $[1,2]$.

*corresponding author; e-mail: stjurga@main.amu.edu.pl 
In this paper we report NMR, dielectric, and rheological studies of metallocene catalyzed ethylene-norbornene copolymer with repeat unit as shown in Fig. 1. The aim of the work is to investigate molecular dynamics of linear copolymer containing ethylene and norbornene units in wide frequency and temperature ranges.

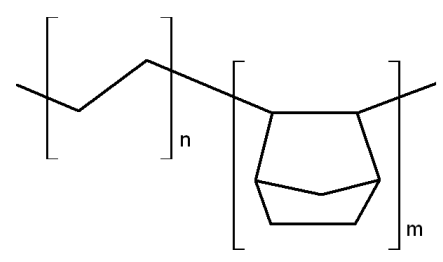

Fig. 1. Chemical structure of ethylene-norbornene copolymer.

The relatively high glass transition temperature of ethylene-norbornene copolymers (up to $180^{\circ} \mathrm{C}$ ) can be altered linearly with increasing the norbornene (NB) comonomer content $[3,4]$. The structure of these copolymers has been extensively studied using ${ }^{13} \mathrm{C}$ high resolution in solution [5-7]. It was found that in copolymer with low NB content the close vicinity of two or more NB groups is rather rare.

\section{Experimental}

The material used in this study is commercially available polymer of brand name Topas ${ }^{\circledR}$ (thermoplastic olefin polymer of amorphous structure) produced by Ticona (Germany). The samples were used in the form of pellets as received without any purification. According to Poulsen et al. [8] the studied sample Topas 8007 has 35 mol percentage of randomly distributed NB.

Differential scanning calorimetry measurements were done using Netzsch 204 Phoenix apparatus. Two cycles were performed, each consisting of heating and cooling of the sample with the rate $20 \mathrm{~K} / \mathrm{min}$. Glass temperature specified from the second heating cycle was of $353 \mathrm{~K}$.

${ }^{1} \mathrm{H}$ NMR experiments were made for samples sealed under vacuum in glass tubes after air evacuation for few hours. Broad-line measurements were carried out with a home-made marginal oscillator spectrometer operating at $28 \mathrm{MHz}$. Second moment values were calculated by numerical integration of spectra and corrected for finite modulation amplitude. Spin-lattice relaxation times $T_{1}$ and $T_{1 \rho}$ were measured at $200 \mathrm{MHz}$ (BRUKER CXP spectrometer) and additional $T_{1}$ measurements were performed at $30 \mathrm{MHz}$ (home-made spectrometer). $T_{1}$ values were determined with saturation recovery technique [9], while $T_{1 \rho}$ by spin-locking the magnetization in the rotating field (of $68 \mathrm{kHz}$ ) [10]. Temperature of the sample was controlled using a conventional gas flow system and monitored with accuracy $1 \mathrm{~K}$. 
Dielectric and mechanical spectroscopies measurements were carried out on films obtained using a SPECAC hot-press system. The material was heated up $50^{\circ} \mathrm{C}$ above the glass transition, held $3 \mathrm{~min}$ to reach a suitable plastic state and pressed using 2 tons load during the next $3 \mathrm{~min}$. Films were quenched in water cooled plates. Measurements of complex permittivity and loss tangent of copolymer that covered the frequency range $10^{-2}$ to $3 \times 10^{6} \mathrm{~Hz}$ and temperature interval 138 to $398 \mathrm{~K}$ ( $5 \mathrm{~K}$ step) were performed with the dielectric spectrometer BDS-80 (Novocontrol) using BDC-S (Alpha Analyzer) probe head. The gold-plated sample of $150 \mu \mathrm{m}$ thickness was kept between electrodes of $20 \mathrm{~mm}$ diameter.

The study of viscoelastic properties was made in oscillatory mode by means of the rheometer ARES (Rheometric Scientific). The complex elasticity modulus and loss tangent of copolymer were determined for 10 frequencies ranged from 0.03 to $15 \mathrm{~Hz}$ in temperature range from 128 to $375 \mathrm{~K}$.

\section{Results and discussion}

The temperature dependence of ${ }^{1} \mathrm{H}$ NMR line second moment $M_{2}$ is shown in Fig. 2. There are three regions where a decrease in the second moment is observed. The first one from 100 to $295 \mathrm{~K}$ with $M_{2}$ dropping from 0.23 to $0.167 \mathrm{mT}^{2}$, the second from 330 to $350 \mathrm{~K}$, where $M_{2}$ decreases to $0.148 \mathrm{mT}^{2}$, and third region above $360 \mathrm{~K}$ where fast decrease to almost zero value appears. Such temperature behavior of the second moment indicates the existence of three different motional processes. The two first reductions of second moment similarly as in polyethylene [11] are connected with local motions, which involve (i) ethylene groups (e.g. trans-gauche isomerization) and (ii) short segments containing aliphatic fragments and norbornene units, respectively. The third one appearing just above glass transition temperature involves large scale rotational and translational motion (liquid-like motion).

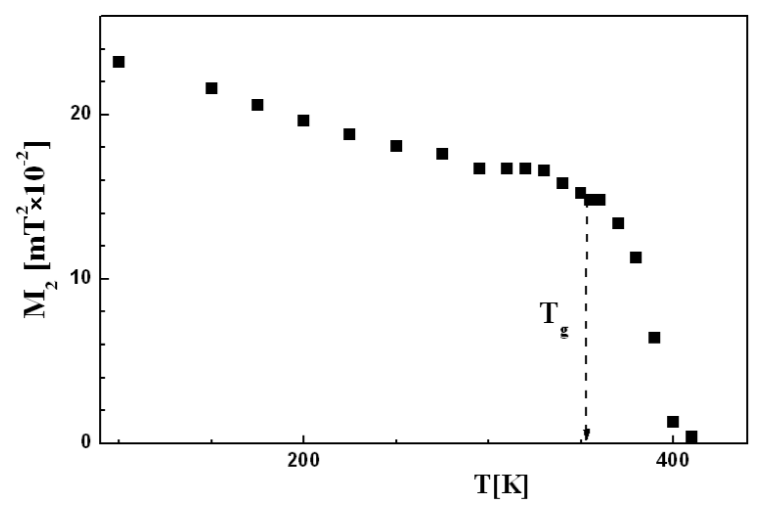

Fig. 2. Temperature dependence of the second moment of ${ }^{1} \mathrm{H}$ NMR line of ethylene-norbornene copolymer. Glass transition temperature is denoted by dashed line. 
Molecular motions in studied copolymer are reflected in measurements of proton spin-lattice relaxation $T_{1}$ and $T_{1 \rho}$ shown in Fig. 3. The observed minima for $T_{1}$ measured at $60 \mathrm{MHz}$ (at $222 \mathrm{~K}$ ) and $T_{1 \rho}$ (at $192 \mathrm{~K}$ ) indicate that reorientational motion takes place. The temperatures at which the minima appear are consistent with gradual decrease in the second moment observed at the lowest temperatures indicating that both features result from the same kind of motion. The qualitative analysis of the spin-lattice relaxation behavior was based on the assumption that temperature dependences of $T_{1}$ and $T_{1 \rho}$, which are governed by dipolar interaction modulated by motional processes, can be approximated by [12]:

$$
\begin{aligned}
\frac{1}{T_{1}} & =\frac{2}{3} \gamma^{2} \Delta M_{2}[J(\omega)+4 J(2 \omega)], \\
\frac{1}{T_{1 \rho}} & =\frac{2}{3} \gamma^{2} \Delta M_{2}\left[\frac{3}{2} J\left(2 \omega_{1}\right)+\frac{5}{2} J(\omega)+J(2 \omega)\right],
\end{aligned}
$$

where $\Delta M_{2}$ is defined as part of the second moment averaged by considered motion, and $J$ 's denote the spectral densities of the correlation functions for the appropriate frequencies. The broad and asymmetrical shapes of $T_{1}$ and $T_{1 \rho}$ minima, as well as their relatively large values reveal that the description of molecular motion with the use of a simple BPP model [13] is inadequate. The experimental data were fitted according to the Havriliak-Negami function [14], for which $J(\omega)$ is defined as follows [15]:

$$
\begin{gathered}
J_{\mathrm{HN}}(\omega)=\frac{2}{\omega} \sin \left\{\varepsilon \arctan \left[\frac{(\omega \tau)^{\delta} \sin (\delta \pi / 2)}{1+(\omega \tau)^{\delta} \cos (\delta \pi / 2)}\right]\right\} \\
\times\left[1+2(\omega \tau)^{\delta} \cos \left(\frac{\delta \pi}{2}\right)+(\omega \tau)^{2 \delta}\right]^{-\varepsilon / 2},
\end{gathered}
$$

where the parameter $\delta$ is a measure of correlations of motions and the product of $\delta$ and $\varepsilon$ describes distribution of correlation times, while $\tau$ is a correlation time, whose temperature dependence was approximated by the Arrhenius equation. The fitting of experimental data was performed for $\Delta M_{2}$ giving the best agreement to $T_{1}$ data. The obtained fits, shown as solid lines in Fig. 3, were obtained for the activation energy of $27 \mathrm{~kJ} / \mathrm{mol}$. Distinct difference between theoretical and experimental value of $T_{1 \rho}$ at minimum may be due to temperature dependence of parameter describing distribution of correlation times and/or the increase in number of ethylene groups involved in the motion when temperature rises up, which was observed in other polymers [11, 16-18].

Motional behavior of the studied copolymer observed in NMR is reflected in dielectric and mechanical studies. The temperature dependences of dielectric and mechanical loss tangent for different frequencies are shown in Fig. 4 and Fig. 5. Three relaxation processes labelled $\gamma, \beta$, and $\alpha$ in order of increasing temperature are evidenced, however in the case of mechanical data the $\beta$ process is revealed 


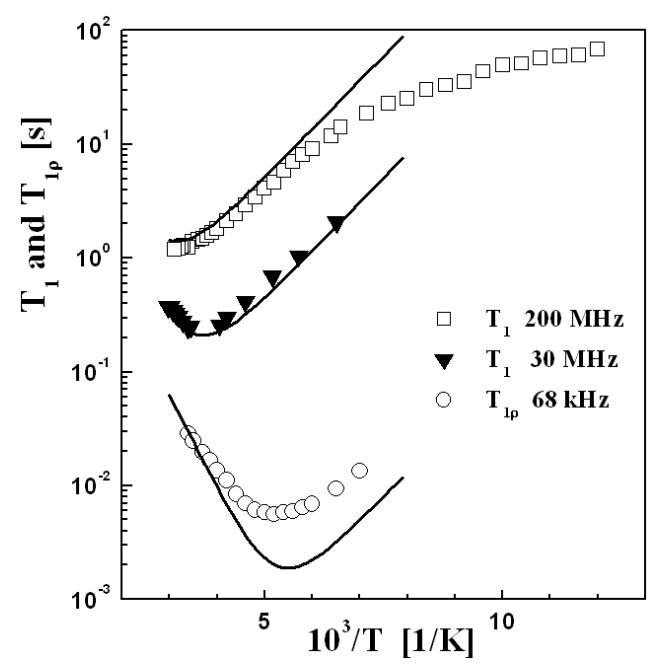

Fig. 3. Temperature dependences of the ${ }^{1} \mathrm{H}$ spin-lattice relaxation times $T_{1}$ and $T_{1 \rho}$ of ethylene-norbornene copolymer. The solid lines represent fits according to the HavriliakNegami function.

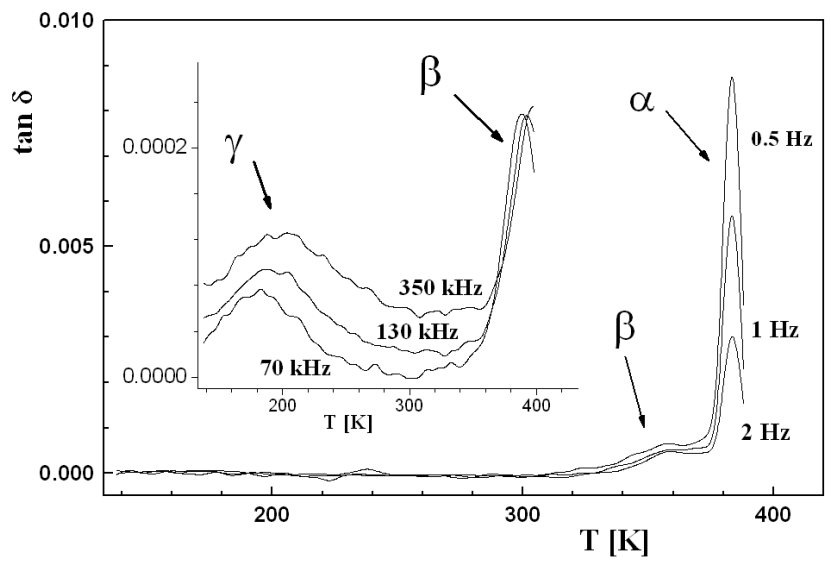

Fig. 4. Temperature dependence of dielectric loss tangent at selected frequencies (main plot - lower frequencies, inserted plot - higher frequencies) for ethylene-norbornene copolymer.

only as a very weak shoulder on the low temperature side of the $\alpha$ process. Taking into account the temperatures and frequencies at which the particular relaxation processes appear it can be stated that these relaxation processes originate from three types of motions, which were recognized in the second moment analysis, namely $\gamma$ process reflecting the local motion of ethylene groups, $\beta$ process resulting from short segmental motions, and $\alpha$ process associated with the glass transition motions. 


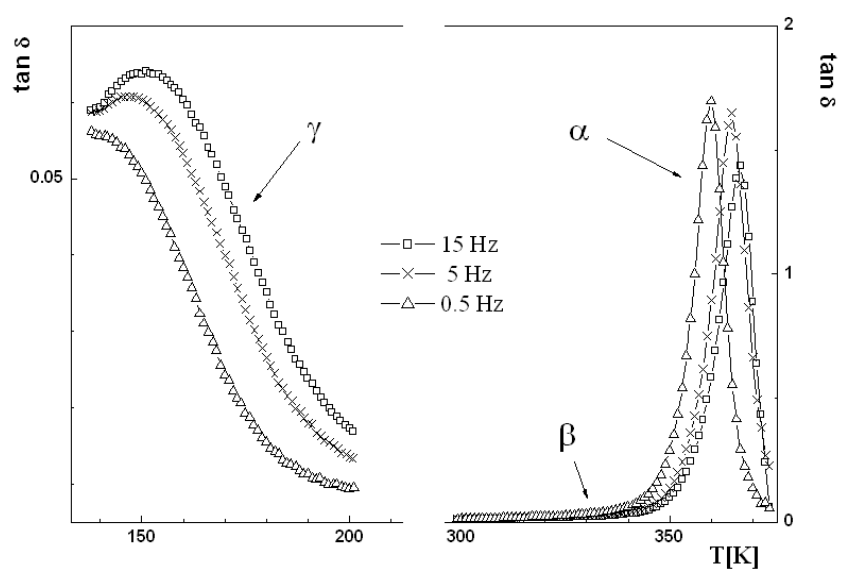

Fig. 5. Temperature dependence of mechanical loss tangent at selected frequencies for ethylene-norbornene copolymer.

The activation energies of considered motions obtained from analysis of frequency-temperature location of the loss maxima together with that obtained from fitting of NMR relaxation data are summarized in Table I.

TABLE I

The activation energies of considered motions. The parentheses involve the temperature ranges for which activation energies were derived.

\begin{tabular}{c|c|c}
\hline \hline & $E_{\mathrm{a}}[\mathrm{kJ} / \mathrm{mol}], \alpha$ process & $E_{\mathrm{a}}[\mathrm{kJ} / \mathrm{mol}], \gamma$ process \\
\hline NMR & - & $27(130 \div 330 \mathrm{~K})$ \\
$\mathrm{DS}$ & $380(364 \div 393 \mathrm{~K})$ & $31(146 \div 200 \mathrm{~K})$ \\
$\mathrm{MS}$ & $533(355 \div 367 \mathrm{~K})$ & $64(128 \div 143 \mathrm{~K})$
\end{tabular}

The obtained values are in good agreement with those derived by Poulsen et al. studies of oxygen diffusion [8] and by Scrivani et al. from dynamic-mechanical thermal analysis (DMTA) measurements [19].

The decrease in the apparent activation energy values for $\alpha$ and $\gamma$ processes for higher frequency window suggests that the rate of motion for these processes can be described by the Vogel-Fulcher-Tammann (VFT) formula [20]:

$$
\frac{1}{\tau}=\frac{1}{\tau_{0}} \exp \left(-\frac{D T_{0}}{T-T_{0}}\right)
$$

where $D$ is fragility parameter and $T_{0}$ is temperature corresponding to an infinite relaxation time.

The temperature dependence of motional frequencies derived from NMR, dielectric, and mechanical studies for $\alpha$ and $\gamma$ process are shown in Fig. 6 and 


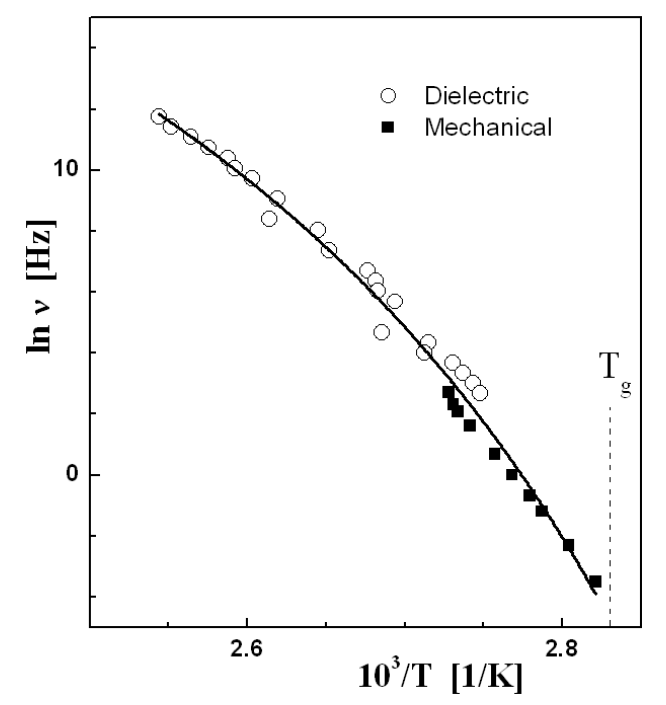

Fig. 6. Relaxation map for $\alpha$ process in ethylene-norbornene copolymer. The solid line represents the best fit according to VFT equation.

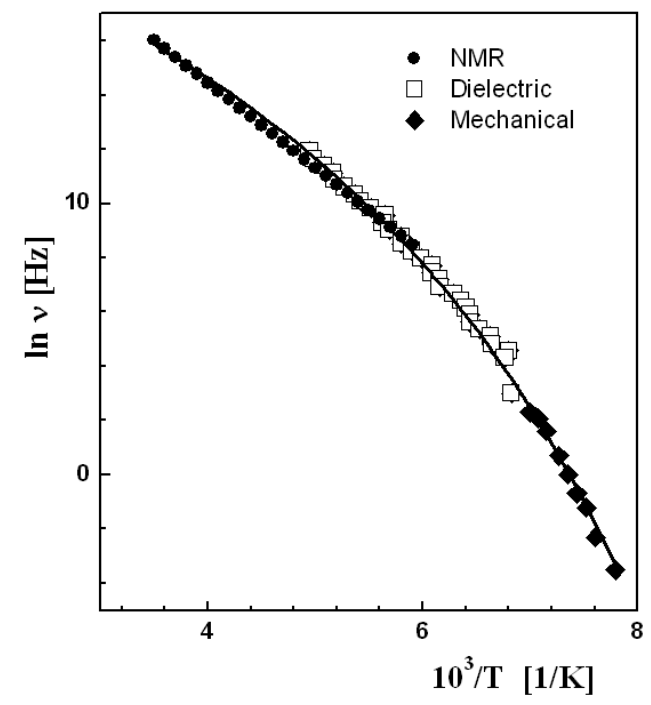

Fig. 7. Relaxation map for $\gamma$ process in ethylene-norbornene copolymer. The solid line represents the best fit according to VFT equation.

Fig. 7, respectively, where the solid lines are fits to VFT equation for parameters presented in Table II.

Good agreement between experimental values and theoretical fits indicates that the motions responsible for $\alpha$ and $\gamma$ processes are cooperative in nature. For $\alpha$ process the cooperative motion is well established phenomenon and can be imag- 
TABLE II

Parameters of VFT fit for $\alpha$ and $\gamma$ processes.

\begin{tabular}{c|c|c}
\hline \hline & $\alpha$ process & $\gamma$ process \\
\hline$D$ & 6 & 15 \\
$T_{0}[\mathrm{~K}]$ & 310 & 80
\end{tabular}

ined as a move of selected segments with its environment. In the case of the $\gamma$ process it seems that the local trans-gauche conformational transitions of methylene groups correlate with motion of next nearby unit, leading also to the non-Arrhenius relaxation. Such behavior was suggested for low temperature relaxation in polymers in molecular dynamics simulation studies [21, 22]. Comparison of fragility parameters for the two processes indicates that the low temperature one is less cooperative than that connected with glass transition.

At this stage of study we have not precisely analyzed the motional parameters of $\beta$ process. We want only to mention that peak intensity depends on thermal treatment of sample which was also observed by Chu et al. [23].

\section{Conclusions}

NMR, dielectric, and mechanical spectroscopies were used to characterize in a broad frequency range the molecular dynamics of ethylene-norbornene copolymer. Three motional processes, denoted as $\alpha, \beta$, and $\gamma$ are detected. The $\gamma$ and $\beta$ processes are associated with local motion. The results show that $\gamma$ relaxation has similar origin to that of polyethylene trans-gauche isomerization, while $\beta$ process results from short range segmental motion in which norbornene units are involved. The $\alpha$ process is attributed to the large scale motions associated with the dynamic glass transition. Temperature dependences of frequencies of motion for $\alpha$ and $\gamma$ relaxations are well described by the Vogel-Fulcher-Tammann equation indicating cooperative nature of the molecular motions.

\section{Acknowledgments}

A partial support of 6th Framework Programme under SoftComp grant No. 502235-2 is gratefully acknowledged.

\section{References}

[1] R.R. Lamonte, D. McNally, Plastic Eng. 56, 51 (2000).

[2] R. Mülhaupt, Macromol. Chem. Phys. 204, 289 (2003). 
[3] G.M. Benedikt, E. Elce, B.L. Goodall, H.A. Kalamarides, L.H. III McIntosh, L.F. Rhodes, K.T. Selvy, C. Andes, K. Oyler, A. Sen, Macromolecules 35, 8978 (2002).

[4] K. Thorshaug, R. Mendichi, L. Boggioni, I. Tritto, S. Trinkle, C. Friedrich, R. Mülhaupt, Macromolecules 35, 2903 (2002).

[5] M. Arndt-Rosenau, I. Beulich, Macromolecules 32, 7335 (1999).

[6] I. Tritto, C. Marestin, L. Boggioni, M.C. Sacchi, H.-H. Brintzinger, Macromolecules 34, 5770 (2001).

[7] I. Tritto, C. Marestin, L. Boggioni, L. Zetta, A. Provasoli, D.R. Ferro, Macromolecules 33, 8931 (2000).

[8] L. Poulsen, I. Zebger, M. Klinger, M. Eldrup, P. Sommer-Larsen, P.R. Ogilby, Macromolecules 36, 7189 (2003).

[9] E. Fukushima, S.B.W. Roeder, Experimental Pulse NMR, Addison-Wesley, London 1981, p. 174.

[10] D.C. Look, I.J. Lowe, J. Chem. Phys. 44, 2995 (1966).

[11] S. Glowinkowski, M. Makrocka-Rydzyk, S. Wanke, S. Jurga, Eur. Polym. J. 38, 961 (2002).

[12] E.R. Andrew, J.M. Radomski, Solid State Nucl. Magn. Reson. 2, 57 (1993).

[13] N. Bloembergen, E.M. Purcell, R.V. Pound, Phys. Rev. 73, 679 (1948).

[14] Jr.S. Havriliak, S. Negami, J. Polym. Sci. C 14, 99 (1966).

[15] P.A. Beckmann, Phys. Rep. 3, 85 (1988).

[16] M.S. Graff, R.H. Boyd, Polymer 35, 1797 (1994).

[17] G. Floudas, P. Placke, P. Stepanek, W. Brown, G. Fytas, K.L. Ngai, Macromolecules 28, 6799 (1995).

[18] D. Hentschel, H. Sillescu, H.W. Spiess, Polymer 25, 1078 (1984).

[19] T. Scrivani, R. Benavente, E. Pérez, J.M. Pereña, Macromol. Chem. Phys. 202, 2547 (2001).

[20] H. Vogel, Phys. Z. 22, 645 (1921); G.S. Fulcher, J. Am. Chem. Soc. 8, 339 (1925); G. Tammann, G. Hesse, Annorg. Allg. Chem. 156, 245 (1926).

[21] Y. Jin, R.H. Boyd, J. Chem. Phys. 108, 9912 (1998).

[22] M. Fukuda, H. Kikuchi, J. Chem. Phys. 113, 4433 (2000).

[23] P.P. Chu, M.-H. Cheng, W.-J. Huang, F.-C. Chang, Macromolecules 33, 9360 (2000). 